\title{
Alleles Frequency and Polymorphic Genes Genotypes Associated with Alcoholism in Kazakh Population
}

\author{
Svetlana Kaliullaevna Sakhanova (iD ${ }^{1}$, Gulnara Salavatovna Svyatova (iD ${ }^{2}$, Valery Petrovich Pavlenko (iD) ${ }^{3}$, \\ Akmaral Adietovna Ramazanova (iD ${ }^{3}$, Stanislav Vladimirovich Zaynullin (iD ${ }^{3}$ and Daniya Smagulova \\ (iD) 4 , * \\ ${ }^{1}$ Scientific-Practical Center, West Kazakhstan Marat Ospanov Medical University, Aktobe, Kazakhstan \\ ${ }^{2}$ Scientific Center of Joint-Stock Company “Scientific Center of Obstetrics, Gynecology and Perinatology”, Almaty, Kazakhstan \\ ${ }^{3}$ West Kazakhstan Marat Ospanov Medical University, Aktobe, Kazakhstan \\ ${ }^{4}$ Department of Natural Science Disciplines, West Kazakhstan Marat Ospanov Medical University, Aktobe, Kazakhstan \\ "Corresponding author: Department of Natural Science Disciplines, West Kazakhstan Marat Ospanov Medical University, Aktobe , Kazakhstan Email: \\ smagulovadaniya@gmail.com \\ Received 2021 May 21; Revised 2021 July 14; Accepted 2021 August 07.
}

\begin{abstract}
Background: There is a category of people with a congenial predisposition to alcohol abuse among the total population. The identification of such persons by molecular genetic diagnostics and the implementation of appropriate preventive measures can significantly reduce the incidence of alcoholism.

Objectives: This research aimed to study the genetic foundations of alcohol dependence development in Kazakhs based on the analysis of population frequencies of polymorphic variants of predisposition to alcoholism genes.

Materials and Methods: The material for the research was the DNA recovered from the peripheral blood of the recruited control group population, which was represented by 1,800 conditionally healthy individuals of Kazakh nationality. Isolated DNA samples were genotyped by PCR.

Conclusions: Kazakhs take an intermediate position between the previously studied European and Asian populations by allele frequencies of nine polymorphic variants of $A D H 1 B$ (rs2066701, rs1789891), ADH1C (rs1693425, rs698), HTR2C (rs6318), ALDH2 (rs671), CADM2 (rs9841829), KLB (rs11940694), DRD2 (rs1076560) genes. Possible markers of an increased risk of alcoholism development in Kazakhs are G alleles of polymorphic loci rs2066701 of the $A D H 1 B$ gene and rs671 of the ALDH2 gene, and the protective effect is possible in the presence of A alleles rs2066701 of the $A D H 1 B$ gene and rs671 of the $A L D H 2$ gene.
\end{abstract}

Keywords: GWAS, Predisposition to Alcoholism Development, Genetic Markers, Molecular Genetic Research, Alcoholism

\section{Background}

The genetic factors play an important role at the basis of the formation of alcoholism and reflect the individual characteristics of neurotransmitter systems activity and enzymes of alcohol metabolism. According to the World Health Organization, alcohol abuse involves the deaths of 2.5 million people worldwide every year. By alcohol consumption, Kazakhstan is accounted for 34th out of 188 countries of the world (10.96 liters of alcohol per capita) and first place among countries of Central Asia (1-3). Due to the growth of the Kazakh population suffered by alcohol, genetic research based on this disease can have a special significance for the development of efficient methods of prevention and pathogenetic therapy $(4,5)$. However, the molecular genetic study of alcoholism has not previously been carried out in the Republic of Kazakhstan. Thus, identifying polymorphic variants in candidate genes that are the most significant in the development of this disease is an urgent challenge for fundamental science and practical medicine.

We investigate the genetic underpinnings of alcohol dependence development in Kazakhs based on population frequencies of polymorphic variations of propensity to alcoholism genes. These genes are selected from global GWAS catalog based on the previous studies of researchers. We calculate and describe the allele frequencies of nine polymorphic variants of genes related to chronic alcoholism. The $A D H 1 B$ gene (Alcohol Dehydrogenase $1 \mathrm{~B}$ (Class I), Beta Polypeptide, rs2066701 (Arg369Cys), and rs1789891 (Arg272Gln)) encodes a protein that is a member of the alcohol dehydrogenase family. These enzymes metabolize ethanol. The $A D H 1 C$ gene (Alcohol Dehydrogenase $1 \mathrm{C}$, 
rs1693425 (HaeIII site), and rs698 (Ile349Val)) encodes the $\gamma$-subunit of the ethanol-metabolizing enzyme. The $A D H 1 B$ and $A D H 1 C$ genes encode the primary ADH enzymes for alcohol metabolism in the liver. The aldehyde dehydrogenase (ALDH- rs671 (Glu504Lys)) superfamily belongs to the APDH gene, which is the primary enzyme responsible for ethanol metabolism in the liver. It encodes the aldehyde dehydrogenase-2 enzyme, which converts acetaldehyde to acetate during detoxification. Potential targets for genetic susceptibility in the pharmacodynamic actions of ethanol are serotonin 5-HT 2 receptors rs6318 (Cys23Ser), dopamine DRD2 receptors D2, rs1076560 ( $>$ A ), etc. Besides, KLB ( $\beta$-Klotho Beta (rs11940694 $(\mathrm{A}>\mathrm{G})$ ) is a transmembrane protein that combines with FGF receptors to form cell surface receptors for the hormones FGF19 and FGF21. One of the significant loci of predisposition to alcoholism is $\beta$ Klotho. Also, CADM2 (cell adhesion molecule 2, (rs9841829 $(\mathrm{T}>\mathrm{G})$ ) is a brain-expressed gene encoding the cell adhesion molecule 2 .

\section{Materials and Methods}

To determine the clinical and diagnostic significance of gene polymorphisms associated with alcoholism in the Kazakh population, a molecular genetic study of their frequency characteristics in the control population was carried out. The DNA samples from 1,800 conditionally healthy individuals of Kazakh nationality were isolated from peripheral blood using the Prepito DNA CytoPureKit and M-PVA magnetic particle separation technology on a Chemagic Prepitto (PerkinElmer) automatic analyzer. Genotyping of $A D H 1 B$ (rs2066701, rs1789891), $A D H 1 C$ (rs1693425, rs698), HTR2C (rs6318), ALDH2 (rs671), CADM2 (rs9841829), KLB (rs11940694), and DRD2 (rs1076560) was performed with Polymerase Chain Reaction (PCR). All the examined people were made aware of the aims of the project and signed an informed consent form to participate.

The conditionally healthy individuals as the control population were 1026 females and 774 males. The average age of the participants in the study at the time of the questionnaire and blood survey was $26.76 \pm 1.61$ years. The results of GWAS were analyzed using global databases including NCBI [http://www.ncbi.nlm.nih.gov], Ensembl [http://asia.ensembl.org/index.html], HapMap [http://hapmap.ncbi.nlm.nih.gov/index.html.en], and 1000 genomes [www.1000genomes.org]. Haploview application was used in the GWAS to analyze and visualize haplotypes and statistical genomics.

The significance of differences in the frequencies of each SNP polymorphism between the main and control groups was calculated using PLINK software (6). Significance for each SNP was statistically assessed by the $\chi^{2}$ method or a similar indicator. The assessment of the conformity of the obtained genotypes frequencies to Hardy-Weinberg equilibrium law was calculated with the HWEtest function of PLINK software. Genetic distances were calculated with PLINK software.

\section{Results and Discussion}

We conducted cross-sectional general population research among healthy Kazakhs and compared the results of genotype and allele frequencies of $A D H 1 B$ (rs2066701, rs1789891), $A D H 1 C$ (rs1693425, rs698), HTR2C (rs6318), ALDH2 (rs671), CADM2 (rs9841829), KLB (rs11940694), and DRD2 (rs1076560) polymorphisms with global populations. We discovered potential markers of alcoholism development in Kazakhs.

Table 1 illustrates the important SNP polymorphisms selected from the worldwide GWAS databases that influence the development of alcoholism, as well as the frequencies of minor alleles in the studied groups of Kazakhs. The lowest population frequency of the minor allele (0.013) was found for the polymorphism of the $A L D H 2$ gene, while the highest frequency was 0.368 for the $K L B$ carriership of allele C. For all investigated polymorphisms in the Kazakh population, the distribution of genotypes conformed to the Hardy-Weinberg equilibrium ( $\mathrm{P}>0.05)$.

In our sample of 1,800 Kazakhs, the frequency of A minor allele rs2066701 polymorphism of the betapolypeptide of alcohol dehydrogenase IB gene was $35.4 \%$, which significantly exceeded the population frequencies of A allele in the populations of Europe (29.7\%), Latin America (14.4\%), Finland (22.0\%), and Bosnia (25.7\%) $(\mathrm{P}<$ 0.05). The frequency of population carriership of A allele rs2066701 ( $A D H 1 B$ gene) polymorphism was significantly lower in Kazakhs than in East Asian populations (79.8\%) and South Asian populations (54.0\%) $(\mathrm{P}<0.001)$.

The frequency of the unfavorable allele A of the $A D H 1 B$ gene for the second rs1789891 polymorphism in the Kazakh groups was $13.1 \%$, which did not differ significantly from the populations of Europe (14.7\%) (P > 0.05), but was significantly lower than the previously published frequencies for the populations of South Asia, Latin America, Finland, and Great Britain $(\mathrm{P}<0.05)(7)$.

For the second gene of the family of alcohol dehydrogenases $A D H 1 C$, the frequency of the minor allele by polymorphism (rs1693425) in the Kazakh population was 23.7\%, which turned out to be significantly higher than a similar indicator of East Asian populations (7.6\%), ( $\mathrm{P}<0.001)$, but was significantly lower than similar allelic frequencies of 
Sakhanova SK et al.

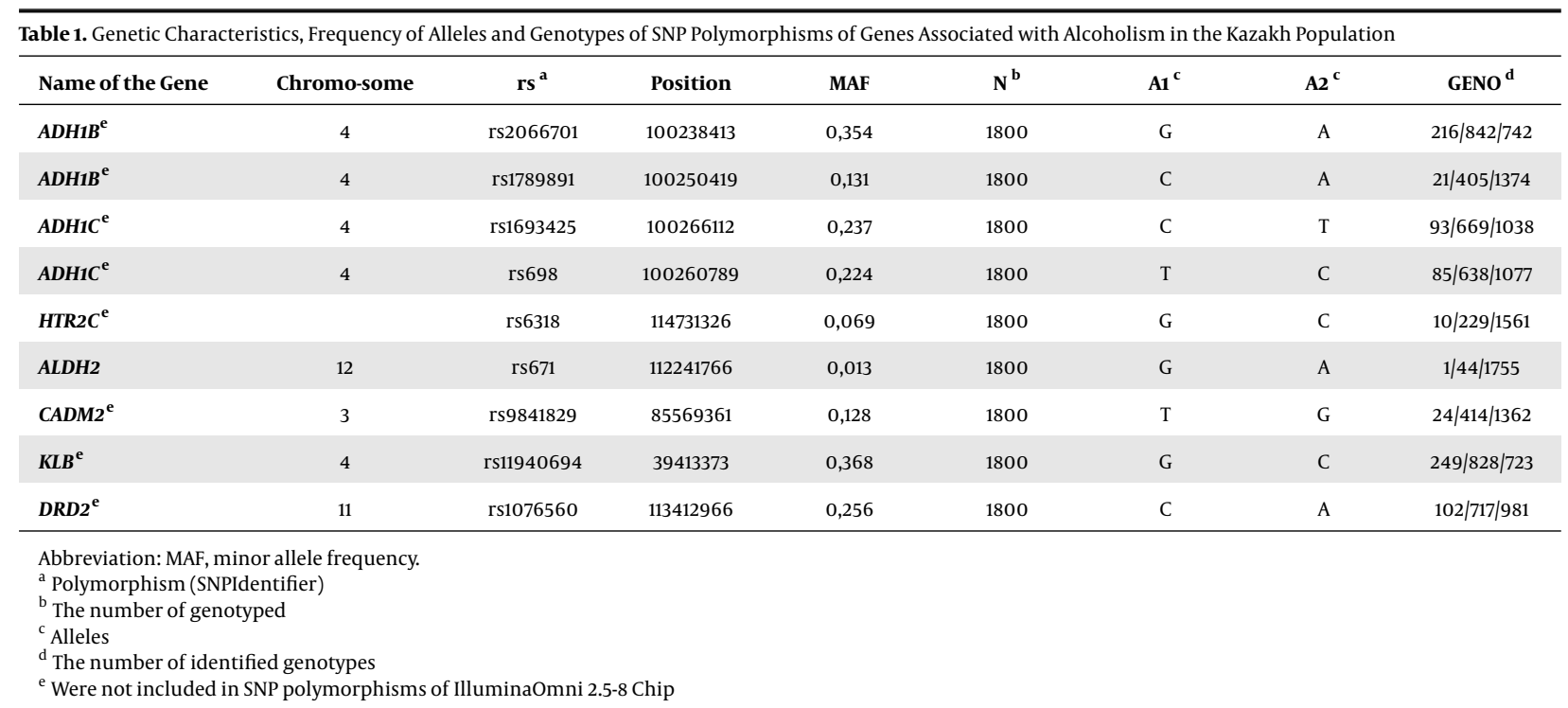

the previously studied populations of Europe, South Asia, Latin America, and Finland $(\mathrm{P}<0.05)$.

The second polymorphism rs698 of the alcohol dehydrogenase $1 \mathrm{C}$ gene demonstrated the population frequency of the minor C allele in Kazakhs as 22.44\%, which significantly exceeded a similar frequency in the populations of East Asia (7.6\%) $(\mathrm{P}<0.001)$, but was significantly lower than all previously studied in the project 1000 genomes of the world's populations ( $\mathrm{P}<0.05)$. The carriership frequency of the protective A allele of rs671 polymorphism of the ALDH2 gene in the Kazakh population was $1.31 \%$, which turned out to be significantly higher than similar frequencies in the African and American populations $(0.2 \%$ and $0.3 \%$, respectively) $(\mathrm{P}<0.001)(8)$. The obtained results coincide with the data that the minor allele of rs6318 polymorphism had a low frequency in Asian populations, China (3.3\%), and Japan (5.7\%).

The carriership frequency of the minor allele $G$ of rs9841829 polymorphism of the CADM2 gene in the Kazakh population showed $12.8 \%$, which turned out to be significantly lower than a similar frequency of its carriership in the populations of Europe, South Asia, Latin America, and Finland $(\mathrm{P}<0.05)$. As we investigated, the population frequency of carriership of the unfavorable allele A of the rs11940694 polymorphism (KLIB gene) in the Kazakh population turned out to be low and made $36.8 \%$, which is comparable to the population of South Asia and Latin America $(P>0.05)$, but was significantly lower than a similar frequency of European population (61.2\%) including Finland (65.7\%) and East Asian population (45.9\%) $(\mathrm{P}<0.001)$.

The frequency of the A allele of rs1076560 polymorphism of DRD2 gene in the Kazakh population was $25.6 \%$, which did not show significant differences with the population of South Asia (28.9\%) (P > 0.05). The carriership frequency of the unfavorable allele A in Kazakhs significantly exceeded its frequency in the populations of Europe (15.0\%) and Finland (20.8\%), but turned out to be significantly lower than that of the indigenous population of East Asia, Latin America, and Pakistan $(\mathrm{P}<0.05)$.

Kazakhs take an intermediate position between the previously studied European and Asian populations by allele frequencies of nine polymorphic variants of $A D H 1 B$ (rs2066701, rs1789891), ADH1C (rs1693425, rs698), HTR2C (rs6318), ALDH2 (rs671), CADM2 (rs9841829), KLB (rs11940694), and DRD2 (rs1076560) genes.

In conclusion, the potential indicators of higher risk of alcoholism in Kazakhs are $\mathrm{G}$ alleles of polymorphic loci rs2066701 of the $A D H 1 B$ gene and rs671 of the $A L D H 2$ gene, with possible protective effects of the A allele of rs2066701 of $A D H 1 B$ and rs671 of ALDH1B gene. At present, only a general population study has been conducted in the Kazakh population compared to the populations of the world. The study of gene polymorphisms in alcoholism patients in the Kazakh population is the next stage of our research.

\section{Footnotes}

Authors' Contribution: GS wrote the initial manuscript draft; SS, VP, and DS arranged the database and improved the manuscript; AR and SZ performed the statistical analysis; All authors were involved in the manuscript improvement, read, and approved the version presented.

Conflict of Interests: The authors have no potential conflicts of interest. 
Ethical Approval: The research work was approved by the Bioethical Committee of the West Kazakhstan Marat Ospanov State Medical University (MoM No. 16 dated 11.04.2019).

Funding/Support: This study was funded by "Marat Ospanov West Kazakhstan Medical University", a noncommercial joint-stock company.

Informed Consent: All the examined people were made aware of the aims of the project and signed an informed consent form to participate.

\section{References}

1. McCarthy MI, Hirschhorn JN. Genome-wide association studies: Past, present and future. Hum Mol Genet. 2008;17(R2):R100-1. doi: 10.1093/hmg/ddn298.

2. Nashkenova A, Zhamalieva D, Zholaushibekova A, Asfendiyarov SD. The prevalence of alcoholism in Kazakhstan (review and interpretation of statistical data). Electronic resource; 2015. Available from: https: //kaznmu.kz/press/wp-content/uploads/2015/.

3. Nature. Genome-wide association study of 14,000 cases of seven common diseases and 3,000 shared controls. Nature. 2007;447(7145):66178. doi: $10.1038 /$ nature05911.

4. Johnson EC, St. Pierre CL, Meyers JL, Aliev F, McCutcheon VV, Lai D, et al. The genetic relationship between alcohol consumption and aspects of problem drinking in an ascertained sample. Alcohol Clin Exp Res. 2019;43, I(6):1113-25. doi: 10.1111/acer.14064.

5. Edenberg HJ, McClintick JN. Alcohol dehydrogenases, aldehyde dehydrogenases, and alcohol use disorders: A critical review. Alcohol Clin Exp Res. 2018;42(12):2281-97. doi: 10.1111/acer.13904.

6. Price AL, Patterson NJ, Plenge RM, Weinblatt ME, Shadick NA, Reich D. Principal components analysis corrects for stratification in genome-wide association studies. Nat Genet. 2006;38(8):904-9. doi: 10.1038/ng1847.

7. Borinskaya S, Kal'ina N, Marusin A, Faskhutdinova G, Morozova I, Kutuev I, et al. Distribution of the alcohol dehydrogenase $\mathrm{ADH} 1 \mathrm{~B} * 47 \mathrm{His}$ allele in Eurasia. Am J Hum Genet. 2009;84(1):89-92. doi: 10.1016/j.ajhg.2008.12.007.

8. National Library of Medicine. rs671, 1000genomes. 2021. 